\title{
A comparative study of fat body morphology in five mosquito species
}

\author{
Gustavo Ferreira Martins ${ }^{1 /+}$, José Eduardo Serrão ${ }^{1}$, \\ José Marcelo Ramalho-Ortigão², Paulo Filemon Paolucci Pimenta ${ }^{3}$ \\ ${ }^{1}$ Departamento de Biologia Geral, Universidade Federal de Viçosa, Viçosa, MG, Brasil \\ ${ }^{2}$ Department of Entomology, Kansas State University, Manhattan, Kansas, USA \\ ${ }^{3}$ Laboratório de Entomologia Médica, Instituto René Rachou-Fiocruz, Belo Horizonte, MG, Brasil
}

\begin{abstract}
The insect fat body plays major roles in the intermediary metabolism, in the storage and transport of haemolymph compounds and in the innate immunity. Here, the overall structure of the fat body of five species of mosquitoes (Aedes albopictus, Aedes fluviatilis, Culex quinquefasciatus, Anopheles aquasalis and Anopheles darlingi) was compared through light, scanning and transmission electron microscopy. Generally for mosquitoes, the fat body consists of lobes projecting into the haemocoel and is formed by great cell masses consisting of trophocytes and oenocytes. Trophocytes are rich in lipid droplets and protein granules. Interestingly, brown pigment granules, likely ommochromes, were found exclusively in the trophocytes located within the thorax and near the dorsal integument of Anopheles, which is suggestive of the role these cells play in detoxification via ommochrome storage. This study provides a detailed comparative analysis of the fat body in five different mosquito species and represents a significant contribution towards the understanding of the structural-functional relationships associated with this organ.
\end{abstract}

Key words: fat body - trophocyte - ommochrome - Aedes - Culex - Anopheles

The fat body is the main storage and intermediary metabolism organ of insects and is responsible for the synthesis and supply of haemolymph substances. It consists of a mass of cells located underneath the epidermis and, in some insects, the fat body also surrounds the digestive and reproductive organs (reviewed by Haunerland \& Shirk 1995 and Roma et al. 2010). Fat body cells, known as trophocytes, are clustered together by a thin basal lamina that expands into the haemocoel and forms amorphous lobes or ribbons that increase the organ surface area, which in turn enhance the exchange of substances between the organ and the haemolymph (Martins \& Pimenta 2008, Arrese \& Soulages 2010).

Trophocytes are mesodermic with a cytoplasm rich in lipids, proteins and glycogen that provide energy and nutrients used mainly for insect locomotion and reproduction (Haunerland \& Shirk 1995, Arrese \& Soulages 2010). Ectodermic cells, such as oenocytes, are also present in the mosquito fat body (Martins et al. 2011c). Oenocytes can be associated with either epidermal cells or trophocytes whose functions are linked with the synthesis of hydrocarbons and homeostasis (Gutierrez et al. 2007, Martins et al. 2011b).

Among blood-sucking insects, mosquitoes are considered the most important disease vectors, with several species involved in the transmission of pathogens such as helminths, protozoans and arboviruses to humans

Financial support: CNPq, FAPEMIG, FIOCRUZ, NIH (AI074691 and AI083831 to JMR-O)

+ Corresponding author: gmartins@ufv.br

Received 19 March 2011

Accepted 17 June 2011 and animals (Eldridge 2004). Despite their importance, many aspects of the physiology of mosquitoes, such as the morphophysiology of the fat body, have been poorly researched. Clearly, the vast number of species encompassing the various mosquito vectors contributes to the difficulty of bridging such knowledge gaps.

The fat body of the yellow fever mosquito Aedes aegypti has been extensively studied with regards to the storage of proteins and lipids used during morphogenesis (Marinotti et al. 2006), the role of lipophorins in lipid transport (Roy et al. 2007), the synthesis of vitellogenin for egg maturation (Roy \& Raikhel 2011) and the detoxification of ammonia from blood meal proteins (Scaraffia et al. 2010). Predominantly, Ae. aegypti has been the focus of recent research on the mosquito's innate immunity (Bian et al. 2005, Feitosa et al. 2006, Antonova et al. 2009). Despite this wealth of information on the basic functions of the fat body, little is known regarding the structural organisation of the fat body in mosquitoes other than Ae. aegypti.

Our recent data on morphological aspects of the $A e$. aegypti fat body revealed the differential staining properties of cells and the fat body's capacity to change at the cellular and subcellular levels according to adult female nutritional status (Martins et al. 2011c). We now extended our investigation to include a comparative structural analysis of the fat body of five adult mosquito species including Aedes albopictus, Aedes fluviatilis, Culex quinquefasciatus, Anopheles aquasalis and Anopheles darlingi. Our approach utilised light microscopy (coupled with histochemistry) along with high magnification scanning and transmission electron microscopy (SEM and TEM) to assess differences in the fat body morphology of these important mosquitoes. Our analyses revealed, for the first time, general and detailed overviews of these mosquito fat bodies. The results obtained 
point to potential significant differences detected in the trophocytes from the tropical anopheline mosquitoes studied, which is suggestive of specific physiological characteristics. In general, trophocytes are rich in nutrient reserves and protein synthesis organelles. However, the trophocytes in the fat bodies of the anophelines studied were also rich in pigment granules, presumably ommochromes. The presence of these pigment granules is discussed regarding their possible role in fat body cell pleomorphism and homeostasis in Anopheles.

\section{MATERIALS AND METHODS}

Mosquitoes - Day-old adult males and females of Ae. albopictus, Ae. fluviatilis, C. quinquefasciatus, An. aquasalis and An. darlingi were used in our study. Except for An. darlingi, all mosquitoes were obtained from colonies maintained at the Laboratory of Medical Entomology of the René Rachou Institute-Fiocruz, Belo Horizonte, Minas Gerais, Brazil. An. darlingi F1 were obtained from field-collected larvae in and around the city of Manaus, Amazonas, Brazil.

Dissection and sample preparation - Mosquitoes were dissected under stereomicroscopy in $300 \mu \mathrm{L}$ of sterile $0.1 \mathrm{M}$ phosphate buffered saline (PBS) pH 7.0. The abdomen was opened longitudinally to expose the fat body attached to the integument. Overall, $25 \mu \mathrm{L}$ of a fixative solution containing 2.5\% glutaraldehyde in PBS supplemented with $4 \%$ sucrose was gently added directly onto the fat body adjacent to the integument. After dissection, this fat body was maintained in the fixative until use in the experiments described below.

SEM - Glutaraldehyde-fixed abdominal fat bodies were post-fixed in $1 \%$ osmium tetroxide in $0.8 \%$ potassium ferrycianide and $0.1 \mathrm{M}$ sodium cacodylate buffer, $\mathrm{pH}$ 7.2. The samples were dehydrated in a crescent series (30-100\%) of acetone, critical-point dried using $\mathrm{CO}_{2}$ and sputter coated with gold for observation under SEM (JEOL JSM 5600).

Light microscopy - For histology and histochemistry, fixed samples were rinsed in PBS, dehydrated in a crescent series of ethanol (70-100\%) and embedded in HistoResin (Leica). Sections ( $2 \mu \mathrm{m}$ thick) were stained with $1 \%$ toluidine blue-borax. Sections were submitted to the following histochemical tests: Gomori's Trichrome for protein staining or periodic acid-Schiff (PAS) for neutral carbohydrates and glucoconjugates. The latter was used only in An. aquasalis and An. darlingi. These methods were adapted from Behmer et al. (1976).

TEM - Abdominal fragments of An. darlingi fat body were transferred to $2.5 \%$ glutaraldehyde in $0.1 \mathrm{M}$ sodium cacodylate buffer, $\mathrm{pH} 7.2$, and post-fixed in $1 \%$ osmium tetroxide plus $0.8 \%$ potassium ferricyanide in the same buffer. Samples were dehydrated in a crescent series of acetone and embedded in Epon-Araldite resin (Electron Microscopy Sciences, EMS, Hatfield, PA). Semi-thin sections were stained with $1 \%$ toluidine blueborax and ultra-thin sections were stained with uranyl acetate and lead citrate. After staining, the ultra-thin sections were washed in distilled water and analysed under TEM (Zeiss EM109).

\section{RESULTS}

SEM - Under SEM, the abdominal fat body of newly emerged adult male and female mosquitoes is an amorphous organ located just underneath the integument. According to its position within the body cavity, the fat body can be divided into dorsal, ventral and lateral portions. The fat body generally has projections (or lobes) that expand from the integument to the visceral organs. Lateral lobes are more prominent than dorsal or ventral lobes, which are hidden by abdominal pleura and muscles (Fig. 1A). The fat body lobes are covered by a thin basal lamina with a sinuous surface. The irregular aspect of the basal lamina is likely due to the presence of fat body cells differently positioned immediately below the lamina (Fig. 1B, C). In some preparations, the basal lamina is eventually broken, exposing cells in the interior of the organ where two types of cells can be recognised. The most common cells, i.e., trophocytes, are globular-shaped with an irregular surface and the oenocytes, which are found less frequently, are smaller and distributed on the fat body surface (Fig. 1D). Interestingly, only the cells of the fat body from both An. aquasalis and An. darlingi display spherical protrusions (Fig. 1E) that are detected in trophocytes located on the fat body periphery in the histological sections. These granules are described in more detail below and are assumed to be ommochrome pigment granules.

Light microscopy - Confirming the SEM results, histological sections also indicated that in newly emerged mosquitoes the fat body is formed by a cell mass underneath the integument with varying thickness along the body cavity and that trophocytes and oenocytes are the two cell types distinctly recognised (Fig. 2A). The trophocytes are morphologically uniform, displaying a spherical shape and a pleomorphic nucleus with the cytoplasm squeezed among many cell inclusions, such as lipid droplets and protein granules that tested positive for the Gomori's Trichrome test (Fig. 2A, B). In contrast, the oenocytes have a large central nucleus and a more homogeneous cytoplasm stained for proteins. Although oenocytes can be found in close proximity to each other or isolated by the trophocytes, they are preferentially located in the periphery of the organ (Fig. 2A).

All species of mosquitoes analysed show the same general organisation and distribution of the fat body cell types. However, fat bodies from the Neotropical anopheline species included in our study (An. aquasalis and An. darlingi) appear distinct based on cytoplasm content from the fat bodies of the two Aedes and C. quinquefasciatus. One of the striking differences is the presence of brown pigment granules found only within trophocytes of the anopheline mosquitoes, which are located primarily around the nucleus and in the cytoplasm periphery (Fig. 2B). The amount of such pigment granules varies between trophocytes. For example, the cytoplasm of trophocytes located in the thorax is almost entirely composed of these granules (Fig. 2C). Additionally, in the dorsal part of the abdomen, near the integument and the dorsal vessel, the pigment granules are more abundant than in the cells from the fat body periphery (i.e., near the haemocoel) (Fig. 2D). 

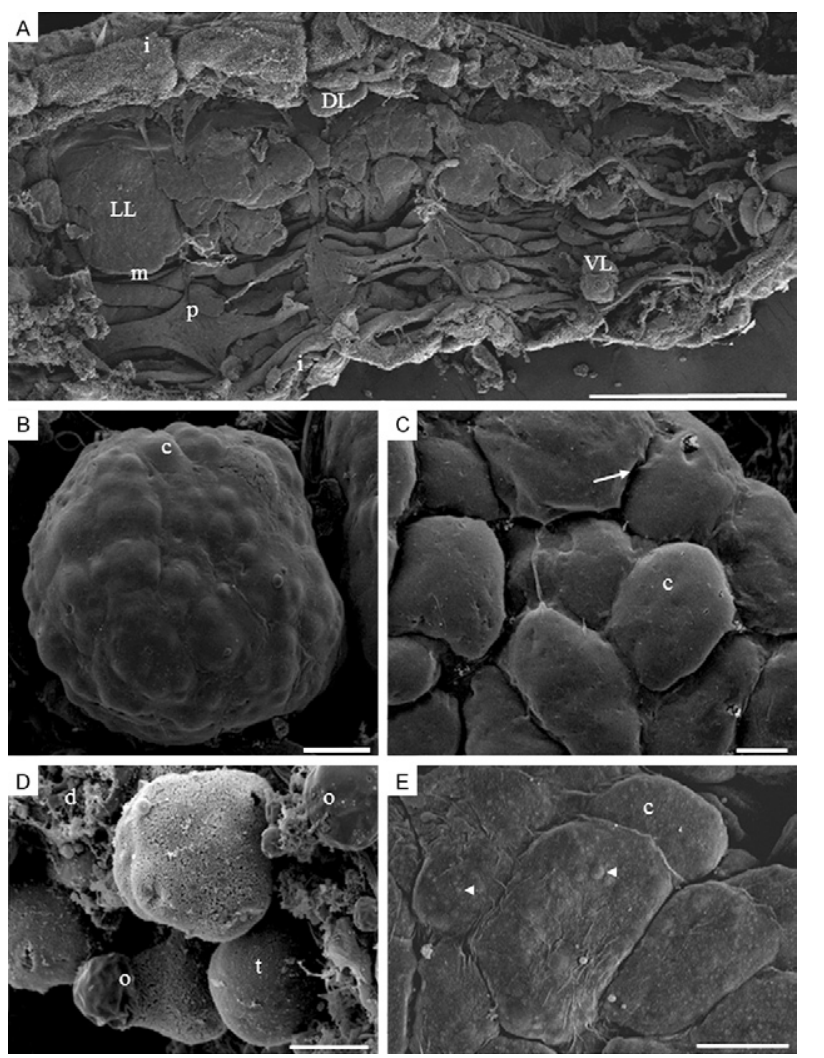

Fig. 1: scanning electron microscopy micrographs showing the general and the detailed views of the fat bodies of newly emerged mosquitoes. A: general view of the opened abdomen of Anopheles aquasalis male displaying the lateral (LL), dorsal (DL) and ventral (VL) fat body lobes (i: integument; m: intersegmental muscle; p: pleura) (Bar= $360 \mu \mathrm{m})$; B: a VL of Culex quinquefasciatus male with irregular surface reflecting the globular shape of the fat body cells (c) inside the lobe $(\mathrm{Bar}=25 \mu \mathrm{m}) ; \mathrm{C}$ : detail of a fat body lobe of Aedes albopictus female displaying cellular profiles of fat body c due the tightness of the basal lamina (arrow) (Bar $=20 \mu \mathrm{m})$; D: detailed view of a fat body lobe of $C$. quinquefasciatus male which had the basal lamina broken showing cells (d: cell debris; o: peripheral oenocytes; t: trophocytes) $(\mathrm{Bar}=10 \mu \mathrm{m})$; E: detail of a fat body lobe of An. aquasalis female displaying cellular profiles of fat body $\mathrm{c}$ and protuberances (arrowheads) that resemble cell inclusions $(\mathrm{Bar}=10 \mu \mathrm{m})$.

To investigate the composition of the brown pigment granules, samples from the abdominal fat body of $A n$. darlingi were also submitted to PAS test. In general, the fat body as a whole test positive, which is contradictory to the result obtained for the brown pigment granules, which are negative for both PAS and the Gomori's Trichrome staining (Fig. 2B, D).

TEM of the An. darlingi female fat body - Considering the difference in cell cytoplasm content (i.e., pigment granules) between trophocytes of Anopheles and three other mosquito species, fat body samples from the abdomen of An. darlingi were used in TEM analyses to investigate the fine structure of the pigment granules.

TEM analyses also confirmed that trophocytes from newly emerged An. darlingi females are differentiated

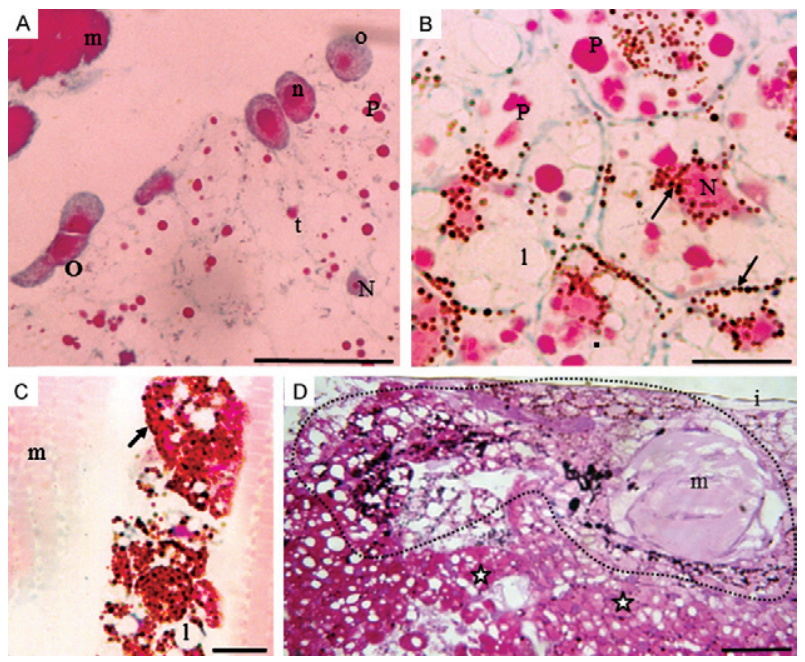

Fig. 2: histological sections of newly emerged mosquito fat bodies. A: Culex quinquefasciatus male fat body stained with Gomori's Trichrome. Trophocytes ( $\mathrm{t}$ ) are the principal cells found in the fat body. Also visible are clustered $(\mathrm{O})$ and isolated (o) oenocytes found mainly in the fat body periphery and uniformly stained for proteins (m: muscle; $\mathrm{n}$ : oenocyte nucleus; N: trophocyte nucleus; P: proteins granules) (Bar $=10 \mu \mathrm{m})$; B: abdominal fat body of Anopheles darlingi female showing $t$ with $\mathrm{P}$ of various sizes. Brown pigment granules found around nucleus and in the cell periphery are indicated by arrows (1: lipid droplets) $(\operatorname{Bar}=15 \mu \mathrm{m})$; $\mathrm{C}$ : thoracic fat body of An. darlingi female. $\mathrm{t}$ are almost completely filled with spherical brown pigment granules (arrow) $(\mathrm{Bar}=10 \mu \mathrm{m})$; D: longitudinal section of the abdominal fat body of Anopheles aquasalis female stained by periodic acid-Schiff test. Notice two different regions, one near abdominal integument (i) rich in $t$ with dark-brown granules (doted line) and another region (stars) with $\mathrm{t}$ displaying fewer granules $(\mathrm{Bar}=60 \mu \mathrm{m})$.

according to location and cell content. In accordance with this finding, cells (trophocytes) located at the fat body periphery predominantly display a cytoplasm filled with lipid droplets and glycogen that have fewer protein granules. The nucleus in these cells is squeezed between cytoplasm inclusions and displays a variable morphology, with an evident nucleolus and regions of condensed chromatin (Fig. 3A). Mitochondria and rough endoplasmic reticulum (RER) are well developed and are detected primarily around the nucleus and beneath the cytoplasmic membrane (Fig. 3B). In contrast, trophocytes near the abdominal integument display a cytoplasm that is poor in RER, mitochondria, lipid droplets and glycogen. In addition, the nucleus is rounded and the chromatin is predominately decondensed. The cytoplasm is rich in granules, which are easily observed under light microscopy, with the smaller spherical electron-dense granules corresponding to pigment granules and the bigger, less electron-dense ones corresponding to protein granules (Fig. 4A).

In the oenocytes, a large number of mitochondria are detected and the cytoplasm is almost entirely filled with well-developed, smooth endoplasmic reticulum (SER) (Fig. 4B). 

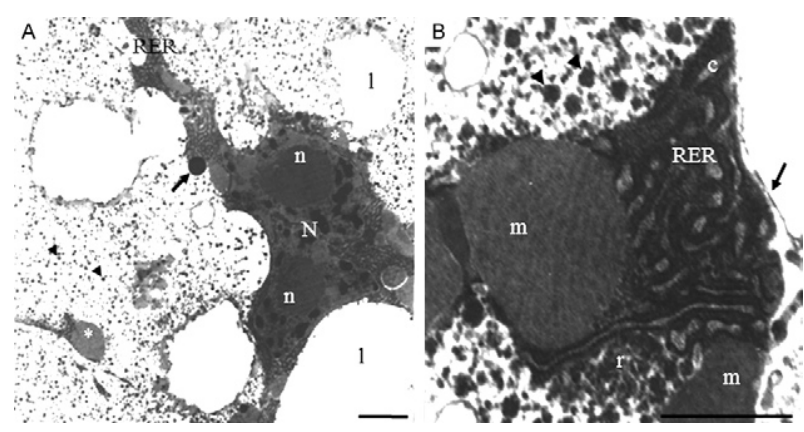

Fig. 3: transmission electron microscopy micrographs of the fat body of newly emerged Anopheles darlingi female showing details of trophocytes. A: lipid droplets (1) and glycogen granules (arrowheads) are abundant. The rough endoplasmic reticulum (RER) is well-developed, extending from nucleus $(\mathrm{N})$ to cytoplasm cortex. $\mathrm{N}$ is squeezed between 1 and has prominent nucleoli (n) (arrow: protein granule) (Bar = $1 \mu \mathrm{m})$; B: detail of a peripheral cytoplasm region containing mitochondria (m), ribosomes (r) and RER with a dilated cistern (c) (arrow: cell membrane; arrowheads: glycogen granules) $(\mathrm{Bar}=0.5 \mu \mathrm{m})$.

\section{DISCUSSION}

The fat body is the most prominent organ in insects and is composed predominantly of trophocytes. However, this simple cell composition does not imply decreased organ metabolism complexity and multitasking ability. For instance, it plays a role in the storage and metabolism of energetic molecules, detoxification, egg yolk precursor synthesis, magnetoreception and immune response (Bian et al. 2005, Feitosa et al. 2006, Roy et al. 2007, Antonova et al. 2009, Cardoso et al. 2010, Roy \& Raikhel 2011). The fat body also actively participates in insect homeostasis by regulating the concentration of substances in the haemolymph (for review see Haunerland \& Shirk 1995, Arrese \& Soulages 2010) and it has also been suggested that it represents a model for a better understanding of metabolism and physiology following cell aging (Hsieh \& Hsu 2011).

Despite the importance of the fat body, only the fat body morphology in the yellow fever mosquito $A e$. aegypti (Raikhel \& Lea 1983, Raikhel 1986a, b, Snigirevskaya et al. 1997, Martins \& Pimenta 2008, Martins et al. 2011c) has been studied in any detail. Our results expand upon the knowledge of fat body organisation in mosquitoes and represent a comparative and comprehensive expansion of the data available on its structural characteristics. To our knowledge, this is the first study to comparatively assess the fat body morphology of other important mosquito vectors such as An. darling (the most important malaria vector in South America), C. quinquefasciatus (the vector of many encephalitis-causing viruses and filariasis) and the Asia tiger mosquito Ae. albopictus (an important vector of several arboviruses, including dengue and yellow fever) (Eldridge 2004).

The SEM analyses showed that the fat body in male and female mosquitoes is an amorphous organ that is not homogenously distributed along the mosquito abdomen. The fat bodies of all mosquitoes are formed by lobes with different sizes that can be subdivided according to their
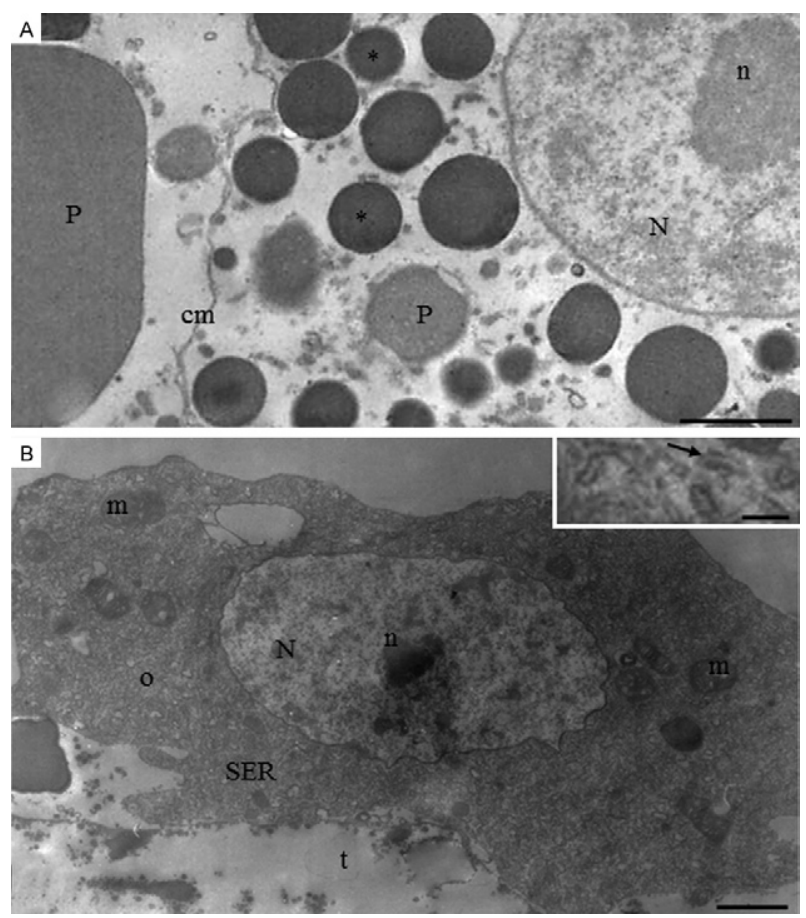

Fig. 4: transmission electron microscopy micrographs of the fat body of newly-emerged Anopheles darlingi female. A: detail of trophocyte cytoplasm rich in granules (somewhat) electron-dense that may refer to protein $(\mathrm{P})$ or brown pigment granules (asterisk) observed in the histological sections (Fig. 2) (cm: cell membrane; n: nucleolus; N: nucleus) $(\mathrm{Bar}=1 \mu \mathrm{m})$; B: general view of an oenocyte $(\mathrm{o})$ in the fat body periphery with well-developed smooth endoplasmic reticulum (SER). Mitochondria (m) are abundant and enlarged [t: trophocyte; inset: tubular SER (arrow) with closed cistern] [Bar $=5 \mu \mathrm{m}$ and $0.3 \mu \mathrm{m}$ (inset)].

location as dorsal, ventral or lateral lobes. Fat body lobes are projected from the body wall towards the visceral organs, supposedly to increase the organ surface area as well as to enhance the exchange between the fat body and the haemocoel. Within the lobes, cell protuberances that increase their contact surface area are also present. Additionally, such patterns of distribution and morphological characters of lobes were previously described in the fat body of Ae. aegypti (Martins \& Pimenta 2008). However, with the current study, we observed that only in Anopheles the basal lamina that lines the fat body have rounded projections that correspond to the pigment granules found in the cytoplasm periphery of the trophocytes.

The cell organisation of the fat body for the adult mosquitoes included in this study is similar to what was reported for Ae. aegypti larvae and adults (Wigglesworth 1942, Martins et al. 2011c), with two easily distinguishable cell types: trophocytes and oenocytes. Under SEM and light microscopy, trophocytes were observed to constitute the main cell population. Trophocyte inclusions represent fat, protein and sugar reserves used by insects to meet their energy demands during reproduction and diapause, to provide energy for the developing embryo and to provide energy for prolonged periods of flight (Arrese \& Soulages 2010). Trophocytes are highly specialised for protein 
synthesis (Raikhel \& Lea 1983, Snigirevskaya et al. 1997, Cardoso et al. 2010) and large amounts of vitellogenic proteins have to be synthesised during mosquito vitellogenesis. Notably, the trophocytes from newly emerged $A n$. darlingi (i.e., in the previtellogenic stage) already display a markedly developed RER (with a dilated cistern), representative of a robust protein synthesis apparatus.

Freshly dissected mosquito abdominal fat bodies are a somewhat yellowish-to-light brown colour, although the two Anopheles included in this study serve as an exception. In An. aquasalis and An. darlingi (and also in the African mosquito Anopheles gambiae), the fat body located near the dorsal vessel is predominantly brown (data not shown). This difference in overall pigmentation may be attributed to the presence of pigment granules inside the trophocytes of Anopheles. According to the results from the Gomori's Trichrome and PAS staining assays, these granules do not contain proteins or neutral polysaccharides and under TEM they appear round and electron-dense. They resemble (in colouration, morphology and distribution inside the cells) ommochrome-containing pigment granules that are present in the central nervous system of the silkworm, Bombyx mori (Sawada et al. 2000), and in the epidermis of dragonflies (Prum et al. 2004) and spiders (Insausti \& Casas 2008, 2009).

Ommochromes are natural organic pigments, especially common in Arthropoda and are usually detected as yellow, red, brown or black pigments. They are synthesised from tryptophan via the kynurenine pathway in Ae. aegypti (Han et al. 2003). Excessive levels of tryptophan are harmful and ommochromes, which can function as eye and integument pigments, as well as excretory products, serve to eliminate excess tryptophan (Tearle 1991). Ommochromes also provide pigmentation for various insect tissues during development (for review see Oxford \& Gillespie 1998, Moraes et al. 2005, Insausti \& Casas 2009). It is known that fat body cells are important for detoxification and homeostasis of insects by recruiting and storing toxic substances from the haemolymph. An association of the fat body with ommochrome synthesis has been reported in larvae of the European corn borer Ostrinia nubilalis (Lepidoptera), where two ommochrome-binding protein genes are transcribed (Coates et al. 2005). It possibly suggests that ommochromes in the fat body play an additional role(s) besides counteracting the toxic effects of tryptophan metabolites. However, the function of ommochromes in the fat body remains to be elucidated.

In contrast to Aedes and C. quinquefasciatus, the trophocytes in Anopheles are pleomorphic. One cell type displays a nucleus with variable morphology and characteristics frequently associated with nutrient synthesis and storage described for Ae. aegypti trophocytes, such as lipid droplets, glycogen granules, RER and mitochondria (Raikhel 1986a, b, Snigirevskaya et al. 1997, Martins et al. 2011c). Another cell type displays a round-shaped nucleus and appears to contain a low number of organelles, such as RER and mitochondria, but is enriched in pigment granules. This latter cell type predominates in the thoracic and dorsal abdominal regions of the fat body in An. aquasalis and An. darlingi.
The oenocytes in all five species studied are located preferentially in the periphery of the fat body and organised in cell clusters or dispersed between trophocytes as previously reported for adult Ae. aegypti (Martins et al. 2011c) and other dipterans (Stoppie et al. 1981, Johnson \& Batterworth 1985). Another common finding is the absence of lipid droplets and glycogen granules in the oenocyte's cytoplasm (Sohal 1973, Tobe et al. 1973, Stoppie et al. 1981). Histochemically, oenocytes have a cytoplasm that is strongly and uniformly stained for proteins, which is evidence of the synthesis of proteins in these cells in the adult mosquito (Wigglesworth 1988, Fan et al. 2003, Roma et al. 2006, Gutierrez et al. 2007, Martins et al. 2011b).

Under the TEM, the principal characteristic of $A n$. darlingi oenocytes is the presence of an enormously extensive SER, as previously reported in Diptera (Sohal 1973, Stoppie et al. 1981), Hemiptera (Wigglesworth 1988), Blattaria (Fan et al. 2003) and Ae. aegypti (Tadkowisk et al. 1977, Martins et al. 2011a). As in other insects, oenocytes in An. darlingi may also be involved in lipid metabolism, including the synthesis of components of a lipidic nature, as suggested by the presence of a well-developed SER (Jackson \& Locke 1989, Fan et al. 2003) and by their gene expression profile in Drosophila melanogaster (Gutierrez et al. 2007) and Ae. aegypti (Martins et al. 2011b).

Underlying the multiple metabolic functions associated with the fat body, the structural pleomorphism of this major insect organ has received limited attention (reviewed in Haunerland \& Shirk 1995). Based on observations in anopheline mosquitoes, the presumption of the mosquito fat body as a homogenous tissue likely overlooks the fact that this organ is more than a simple structure. To date, only those trophocyte histotypes identified in the fat body of adult Anopheles have been investigated. However, future studies focused on biochemical and molecular approaches will help to further elucidate these differences.

Finally, the comparative morphological analyses of the fat bodies in the five mosquito species investigated in this study may provide insight useful for study in other distantly related species. We believe that a comprehensive understanding of fat body morphology in mosquito vectors will contribute to future studies that are focused on structure-function aspects of this vital organ, possibly leading to novel strategies in the fight against such important disease vectors.

\section{ACKNOWLEDGEMENTS}

To the Center for Microscopy and Microanalysis, UFV, for technical assistance.

\section{REFERENCES}

Antonova Y, Alvarez KS, Kim YJ, Kokozak V, Raikhel AS 2009. The role of NF-kB factor REL2 in the Aedes aegypti immune response. Insect Biochem Mol Biol 39: 303-314.

Arrese EL, Soulages JL 2010. Insect fat body: energy, metabolism and regulation. Annu Rev Entomol 55: 207-225.

Behmer OA, Tolosa EMC, Freitas Neto AG 1976. Manual de técnicas para histologia normal e patológica, EDART São Paulo Livraria Editora, São Paulo, 241 pp. 
Bian G, Shin SW, Cheon HM, Kokoza V, Raikhel AS 2005. Transgenic alteration of Toll immune pathway in the female mosquito Aedes aegypti. Proc Natl Acad Sci 102: 13568-13573.

Cardoso AF, Cres RL, Moura AS, de Almeida F, Bijovsky AT 2010. Culex quinquefasciatus vitellogenesis: morphological and biochemical aspects. Mem Inst Oswaldo Cruz 105: 254-262.

Coates BS, Hellmich RL, Lewis LC 2005. Two differentially expressed ommochrome-binding protein-like genes (obpl and obp2) in larval fat body of the European corn borer, Ostrinia nubilalis. J Insect Sci 5: 19.

Eldridge BF 2004. Mosquitoes, the Culicidae. In WC Marquardt, Biology of disease vectors, 2nd ed., Elsevier, p. 95-111.

Fan Y, Zurek L, Dykstra MJ, Schal C 2003. Hydrocarbon synthesis by enzymatically dissociated oenocytes of the abdominal integument of the German cockroach, Blattella germanica. Naturwissenschaften 90: 121-126.

Feitosa FM, Calvo E, Merino EF, Durham AM, James AA, De Bianchi AG, Marinotti O, Capurro ML 2006. A transcriptome analysis of the Aedes aegypti vitellogenic fat body. J Insect Sci 6: 6.

Gutierrez E, Wiggins D, Fielding B, Gould AP 2007. Specialized hepatocyte-like cells regulate Drosophila lipid metabolism. $\mathrm{Na}$ ture 445: 275-280.

Han Q, Calvo E, Marinotti O, Fang J, Rizzi M, James AA, Li J 2003. Analysis of the wild-type and mutant genes encoding the enzyme kynurenine monooxygenase of the yellow fever mosquito, Aedes aegypti. Insect Mol Biol 12: 483-490.

Haunerland NH, Shirk PD 1995. Regional and functional differentiation in the insect fat body. Annu Rev Entomol 40: 121-145.

Hsieh YS, Hsu CY 2011. Honeybee trophocytes and fat cells as target cells for cellular senescence studies. Exp Gerontol 46: 233-240.

Insausti TC, Casas J 2008. The functional morphology of color changing in a spider: development of ommochrome pigment granules. J Exp Biol 211: 780-789.

Insausti TC, Casas J 2009. Turnover of pigment granules: cyclic catabolism and anabolism of ommochromes within epidermal cells. Tissue Cell 41: 421-429.

Jackson A, Locke M 1989. The formation of plasma membrane reticular systems in the oenocytes of an insect. Tissue Cell 21: 463-473.

Johnson MB, Batterworth FM 1985. Maturation and aging of adult fat body and oenocytes in Drosophila as revealed by light microscopic morphometry. J Morphol 184: 51-59.

Marinotti O, Capurro ML, Nirmala X, Calvo E, James AA 2006. Structure and expression of the lipophorin-encoding gene of the malaria vector, Anopheles gambiae. Comp Biochem Physiol 144: 101-109.

Martins GF, Guedes BAM, Silva LM, Serrão JE, Fortes-Dias CL, Ramalho-Ortigão JM, Pimenta PFP 2011a. Isolation, primary culture and morphological characterization of oenocytes from Aedes aegypti pupae. Tissue Cell 43: 83-90.

Martins GF, Pimenta PFP 2008. Structural changes in fat body of Aedes aegypti caused by aging and blood feeding. J Med Entomol 45: 1102-1107.

Martins GF, Ramalho-Ortigão JM, Lobo NF, Severson DW, McDowell MA, Pimenta PFP 2011b. Insights into the transcriptome of oenocytes from Aedes aegypti pupae. Mem Inst Oswaldo Cruz 106: 308-315.

Martins GF, Serrão JE, Ramalho-Ortigão JM, Pimenta PFP 2011c. Hystochemical and ultrastructural studies of the mosquito Aedes aegypti fat body: effects of aging and diet type. Micro Res Tech: doi: $10.1002 /$ jemt 20990.
Moraes AS, Pimentel ER, Rodrigues VLCC, Mello MLS 2005. Eye pigments of the blood-sucking insect, Triatoma infestans Klug (Hemiptera, Reduviidae). Braz J Biol 65: 477-481.

Oxford GS, Gillespie RG 1998. Evolution and ecology of spider coloration. Annu Rev Entomol 43: 619-643.

Prum RO, Cole JA, Torres RH 2004. Blue integumentary structural colours in dragonflies (Odonata) are not produced by incoherent Tyndall scattering. J Exp Biol 207: 3999-4009.

Raikhel AS 1986a. Role of lysosomes in regulating of vitellogenin secretion in the mosquito fat body. J Insect Physiol 32: 597-604.

Raikhel AS 1986b. Lysosomes in the cessation of vitellogenin secretion by the mosquito fat body; selective degradation of Golgi complexes and secretory granules. Tissue Cell 18: 125-142.

Raikhel AS, Lea AO 1983. Previtellogenic development and synthesis in the fat body of a mosquito: an ultrastructural and imunocytochemical study. Tissue Cell 15: 281-300.

Roma GC, Bueno OC, Camargo-Mathias MI 2010. Morpho-physiological analysis of the insect fat body: a review. Micron 41: 395-401.

Roma GC, Camargo-Mathias MI, Bueno OC 2006. Fat body in some genera of leaf-cutting ants (Hymenoptera: Formicidae). Proteins lipids and polysaccharides detection. Micron 37: 234-242.

Roy SG, Hansen IA, Raikhel AS 2007. Effect of insulin and 20-hydroxyecdysone in the fat body of the yellow fever mosquito, $A e$ des aegypti. Insect Biochem Mol Biol 37: 1317-1326.

Roy SG, Raikhel AS 2011. The small GTPase Rheb is a key component linking amino acid signaling and TOR in the nutritional pathway that controls mosquito egg development. Insect Biochem Mol Biol 41: 62-69.

Sawada H, Nakagoshi M, Mase K, Yamamoto T 2000. Occurrence of ommochrome-containing pigment granules in the central nervous system of the silkworm, Bombyx mori. Comp Biochem Physiol 125: 421-428.

Scaraffia PY, Zhang Q, Thorson K, Wysocki VH, Miesfeld RL 2010. Differential ammonia metabolism in Aedes aegypti fat body and midgut tissues. J Insect Physiol 56: 1040-1049.

Snigirevskaya ES, Hays AR, Raikhel AS 1997. Secretory and internalization pathways of mosquito yolk protein precursors. Cell Tissue Res 290: 129-142.

Sohal RS 1973. Fine structural alterations with age in the fat body of the adult male housefly Musca domestica. Z Zellforsch 140: 169-175.

Stoppie P, Briers T, Huybrechts R, De Loof A 1981. Molting hormone juvenile hormone and the ultrastructure of the fat body of adult Sarcophaga bullata (Diptera). Cell Tissue Res 221: 233-244.

Tadkowski TM, Jones JC, Firman J 1977. The fine structure of the imaginal oenocytes of Aedes aegypti. Ann Entomol Soc Am 70: 837-840.

Tearle R 1991. Tissue specific effects of ommochrome pathway mutations in Drosophila melanogaster. Genet Res Camb 57: 257-266.

Tobe SS, Davey KG, Huebner E 1973. Nutrient transfer during the reproductive cycle in Glossina austeni Newst. I. Histology and histochemistry of the milk gland, fat body and oenocytes. Tissue Cell 5: 633-650.

Wigglesworth VB 1942. The storage of protein, fat, glycogen and uric acid in the body and other tissues of mosquito larvae. $J$ Exp Biol 19: 56-77.

Wigglesworth VB 1988. The source of lipids and polyphenols for the insect cuticle: the role of fat body oenocytes and oenocytoids. Tissue Cell 20: 919-932. 